\title{
Index nominum ad Vol. 34
}

Annyas, A. A. 177 (S) Antoni, F. v. Puskás, M. -, - v. Piffko, P. Ami, P. 57 (S) Aschwanden, A. 370 (S) Axelsson, A. and Brorson, J. E. 1

Baumberger, K. 367 (S)

Baumgartner, P. und Hippert, F. 28 (S)

Baumann, R. 36 (S), 369 (S)

Beentjes, B. J. J. 64(B)

Bernath, O. 358 (S)

,$--\mathrm{v}$. Nyffenegger, R.

Bird, E. S. v. Friedmann, J.

Birnmeyer, G. 320(B)

Blattler, W., Northrop, R., Montandon, A.

361 (S) Bleeker, J. D. J. W. 179 (S) Bleuler,K. 42 (S) Böhlau/Böhlau 125 (B) Böhme, G. 372(B)

Boniver, R. v. Penders, C. Bottema, T. 332 (S) Branefors-Helander, P., Nylen, O., and

Jeppson, P. H. 281 Brorson, J. E. v. Axelsson, A. Bruckmann, G. und von Schulthess, G.

366 (S) Buchheim, G. et Sarasin, R. 58 (S) Buiter, C. T. 177 (S), 331 (S)

Cornelissen, M. W. 190 (S) Curry, A. R. v. Daves, J. D. K.

Dammert, K. v. Palva, T.

Dangel, P. v. Novoselac, M.

Dawes, J. D. K. and Curry, A. R. 334 (S)

Dippel,F. 176 (S)

Dobozi, M. 359 (S)

Donat-Jasiak, T. v. Pruszewicz, A.

Edens, E. T. 178 (S)

Eggermont, J. J. v. Odenthal, D. W. -, - and Schmidt, P. H. 355 (S) Escher, F. und Huber, P. 40 (S) -, - $\mathbf{m}$ v. Michel, E. Etholm, B. 273

Farkas, Gy. v. Puskás, M. Feenstra, L. 178 (S) Fisch, U. 58 (S), 359 (S) -, - v. Novoselac, M.

Font, N. 47 (S) Friedmann, G. 41 (S), 336 (S) Friedmann, J., Michaelis, L., Gerwat, J., and Bird, E. S. 195

Gauer, Kramer, Jund 371 (B)

Gerlings, P. G. 181 (S)

Gerwat, J. v. Friedmann, J.

Goumaz, C. F. 39 (S)

Goumoens, R. de 356 (S)

Graf, K. v. Mathis, A.

-, - - v. Zwahlen, H.

Green, A. et Goumoens, R. de 40 (S)

Greve, C. J. 178 (S)

Gundersen, T. 125 (B)

Hadj-Djilani, A. 60 (S) 
Häminnen, P. v. Puhakka, H. J.

Hansson, C. G. and Lindström, J. 82

Haubrich, J. v. Miehlke, A.

Hermon, J. v. Montandon, P.

Hertig, P. 56 (S)

Hippert, F. v. Baumgartner, P.

Hoeksema, P. E. 177 (S), 179 (S)

Höllmüller, O. und Strupler, W. 29 (S)

Hof, E. 371 (S)

Hoshino, T. and Ishii, D. 117

Huber, P. v. Escher, F.

Hürzeler, D. 44 (S)

Huffstadt, A. J. C. 189 (S)

Huygen, P. L. M. v. Marres, E.

Index nominum ad Vol. 34

379

Igarashi, M.. Withers, B. T., and

Miyata, H. 240 Ishii, T. v. Hoshino, T.

Jeppson, P. H. v. Branefors-Helander, P.

John, H. D. v. Westergaard, O.

Jong, P. C. de 303, 333 (S)

-, O. W. de v. Marres, E.

Josuran, J., Vecko, J. und Waidelich, E.

36 (S) Jovanovic, M. 360 (S) Juhn, S. K. v. Youngs, J. N.

Kallenberger, A., Wey, W., and

Renner, K. 65 Kessler, L. und Krisch, A. 165 Kluyskens, P. v. Westergaard, O. Köteles, G. J. v. Pifíko, P. Koopman, T. J. 176 (S) Kornblut, A. D. 325 Krupp, M. A. 320 (B) Kwee, H. L. 145

Lämmli, K. 61 (S), 361 (S) Lehmann, W. 60 (S) -, - et Nicoucar, G. R. 366 (S) Liechti, R. v.

Terrier, G. Lindström, J. v. Hansson, C. G. Loebell, E. 49 (S) Luchsinger und Arnold 64 (B)

Lüönd, E. 30 (S), 360 (S)

Marres, E., Huygen, P. L. M., and Jong,

O. W. de 334 (S) Mathis, A. und Graf, K. 358 (S) Mauerhofer, R. 35 (S) Mehrlin, G. v.

Nyffenegger, R. Meier, D. 54 (S) Meyer, R. 29 (S), 170, 371 (S) Michael, E. 42 (S) Michaelis, L.

v. Friedmann, J. Michel, E. und Escher, F. 359 (S) Miehlke, A., Stennert, E., Schuster, R.,

Schätzle, W. und Haubrich, J. 88 Minnigerode, B. 101 Miyata, H. v. Igarashi, M. Miyoshi, T.

and Pfaltz, C. R. 308

Montandon, P., Hermo, J. et Roth, G.

58 (S) -, A. v. Blattler, W. Montmollin, D. de 38 (S) Moser, F. 191 (B)

Naumann, H. H. 259 (B)

Neiger, M. 357 (S)

,-- v. Nyffenegger, R.

,-- v. Spycher, H.

Nicoucar, G. R. v. Lehmann, W. 366 (S)

Nielsen, B. 210

Northrop, R. v. Blattler, W. 
Novoselac, M., Dangel, P. und Fisch, U.

46 (S) Nyffenegger, R. 51 (S) -, -, Neiger, M., Mehrlin, G. und

Bemath, O. 362 (S) -, - und Bernath, O. 252 Nylen, O. v. Branefors-Helander, P.

Obrebowski, A. v. Pruszewicz, A. Odenthal, D. W. and Eggermont, J. J.

180 (S) Oeken, F. W. 124(B)

Palva, T., Palva, A. and Dammert, K.

153 Penders, C. et Boniver, R. 17 Pfaltz, C. R. 362 (S) -, - v. Miyoshi, T. Piffko, P., Köteles, G.

J., and Antoni, F. 10 Probst, R. 55 (S) Pruszewicz, A., Obrebowski, A., Donat-

Jasiak, T., and Szmegowa, A. 296 Puhakka, H. J. and Hänninnen, P. 220 Puskás, M., Antoni, F.,

Staub, M., and

FarkasGy. 160

Raball, J. A. 356 (S)

Renner, K. v. Kallenberger, A.

Reynier, J. P. de 50 (S)

Rivier, A. v. Savary, M.

Røjskgaer, C. 191 (B)

Rossberg, G. 43 (S)

Rossi, E. 259 (B)

Roth, G. v. Montandon, P.

380

Index nominum ad Vol. 34

Sarasin, R. v. Buchheim, G.

Savary, M. 363 (S)

-, - et Rivier, A. 43 (S), 129, 364 (S)

Schätzle, W. v. Miehlke, A.

Schaller, H. 371 (S)

-, - und Torhorst, J. 38 (S)

Schmidt, P. H. v. Eggermont, J. J.

Schneider, S. v. Terrier, G.

Schnieder, E. A. 227

Schulthess, G. von v. Brückmann, G.

Schuster, E. v. Miehlke, A.

Schweckendieck, W. 259 (B)

Secrétan, J. P. 59 (S), 111, 362

Skouras, J. 367 (S)

Snow, G. B. 180 (S)

Spillmann, T. 357 (S)

Spoendlin, H. 365 (S)

Spycher, H. und Neiger, M. 51 (S)

Staub, M. v. Puskas, M.

Stennert, E. v. Miehlke, A.

Strupler, W. 47 (S)

-, , - v. Höllmüller, O.

Szmejowa, A. v. Pruszewicz, A.

Terrier, G., Schneider, S. et Liechti, R. 
46 (S) Torhorst, J. v. Schaîler, H.

Vecko, J. v. Josuran, J.

Wagner, H. 62 (S) Waidelich, E. v. Josuran, J. Wentges, R. T. R. 332 (S) Wespi, H. H. 33 (S)

Westergaard, O., Kluyskens, P., and

John, H. D. 263 Wey, W. 33 (S), 368 (S) -, n- v. Kallenberger, A. Wille, H. P. 27 (S), 369 (S)

Withers, B. T. v. Iharashi, M. Wolfermann, A. 124(B) Wright, M. I. 124 (B)

Youngs, J. N. and Juhn, S. K. 140

Ziglinas, A. 57 (S), 368 (S) Zwahlen, G., Graf, K. und Vrticka, U. 366 (S) 